\title{
Penjumlahan dari Subnear-ring Fuzzy
}

\author{
Saman Abdurrahman \\ Program Studi Matematika, Fakultas MIPA, Universitas Lambung Mangkurat \\ Л. A. Yani KM 36 Banjarbaru Kalimantan Selatan 70714 \\ Email: samunlam@gmail.com
}

\begin{abstract}
ABSTRAK
Dalam makalah diperkenalkan konsep penjumlahan subnear-ring fuzzy, penjumlahan ideal fuzzy dari nearring, dan membuktikan beberapa sifat dari konsep ini. Hasil dari penelitian ini adalah penjumlahan dari subnearring fuzzy adalah subnear-ring fuzzy, dan penjumlahan ideal fuzzy dari near-ring adalah ideal fuzzy dari near-ring.
\end{abstract}

Kata kunci: jumlah, subnear-ring fuzzy, ideal fuzzy.

\begin{abstract}
This paper introduce the concept on sum of subnear-rings, sum of fuzzy ideals of a near-ring, and prove some properties of this concept. Result of this research is the sum of fuzzy subnear-ring is the fuzzy subnearring, and the sum of fuzzy ideals of near-ring is the fuzzy ideal of near-ring.
\end{abstract}

Keywords: sum, fuzzy subnear-ring, fuzzy ideal.

\section{Pendahuluan}

Penelitian terkait dengan struktur aljabar fuzzy telah banyak dilakukan oleh penelitipeneliti sebelumnya, diantaranya Abou-Zaid [2] memperkenalkan konsep Near-ring fuzzy. Menurut Pilz [6], near-ring merupakan struktur aljabar yang melibatkan dua operasi biner, dimana terhadap operasi pertama membentuk grup yang tidak harus abelian, dan terhadap operasi kedua membentuk semigrup, dan terhadap operasi pertama dan kedua, cukup dipenuhi salah satu sifat distributif kiri atau kanan.

Penelitian near-ring fuzzy selanjutnya dikembangkan pada konsep subnear-ring fuzzy, dan ideal fuzzy dari near-ring. Beberapa penelitian terkait dengan ideal fuzzy dari near-ring telah dilakukan oleh Abdurrahman et al [1] diantaranya adalah ideal fuzzy dari near-ring, yang meliputi hubungan antara ideal dengan ideal fuzzy dari suatu near-ring. Dalam tulisan ini akan disajikan hasil kajian teori tentang penjumlahan subnear-ring fuzzy dari suatu near-ring. Ide tulisan ini dimotivasi oleh pernyataan Satyanarayana et al [7] menyatakan, jika A dan B adalah ideal dari suatu near-ring $\mathrm{R}$, maka $\mathrm{A}+\mathrm{B}$ adalah ideal dari $\mathrm{R}$. Dari sifat ini muncul suatu pertanyaan, apakah sifat ini berlaku juga pada ideal fuzzynya? Berangkat dari permasalahan ini, maka dalam tulisan ini akan dibicarakan hasil penjumlahan dari subnearring fuzzy, yang meliputi penjumlahan ideal fuzzy dari suatu near-ring.

\section{Metode Penelitian}

Penelitian ini dilakukan berdasarkan studi literatur dari berbagai sumber baik berupa buku, atau jurnal ilmiah, khususnya yang berkaitan dengan near-ring, subnear-ring, ideal nearring, near-ring fuzzy, subnear-ring fuzzy, dan ideal fuzzy near-ring.

Pada tahap awal dipelajari konsep-konsep dasar tentang near-ring, subnear-ring, dan ideal near-ring. Konsep dasar ini yang nantinya akan banyak membantu dalam memahami konsep near-ring fuzzy, subnear-ring fuzzy, dan ideal fuzzy near-ring.

Setelah memahami konsep near-ring fuzzy, subnear-ring fuzzy, dan ideal fuzzy near-ring, selanjutnya mendefinisikan penjumlahan dari dua subset fuzzy, membuktikan beberapa lemma atau teorema yang terkait, dan menentukan asumsi-asumsi sehingga terbentuk sifat baru, yang mendukung pada pembuktian hasil penjumlahan antara subnear-ring fuzzy, dan penjumlahan antara ideal fuzzy near-ring. 
Langkah terakhir, dengan menggunakan lemma atau teorema yang saling terkait, maka dibuktikan lemma atau teorema dari hasil penjumlahan antara subnear-ring fuzzy, dan penjumlahan antara ideal fuzzy near-ring.

\section{Hasil dan Pembahasan}

Sebelum membahas hasil dari penjumlahan antara subnear-ring fuzzy, dan penjumlahan antara ideal fuzzy near-ring, berikut diberikan beberapa definisi, lemma, dan teorema yang mendukung pada pembahasan selanjutnya.

Definisi 1. (Pilz, [6]) Himpunan $R$ tidak kosong dengan dua operasi biner + dan . disebut near ring, jika memenuhi:

(1) $(R,+)$ adalah grup (tidak harus grup abelian),

(2) $(R,$.$) adalah semigrup,$

(3) untuk setiap $x, y, z \in \mathrm{R}$ berlaku salah satu sifat distributif kanan atau kiri

(i). distributif kanan : $(x+y) \cdot z=x \cdot z+y \cdot z$

(ii). distributifkiri $: x \cdot(y+z)=x \cdot y+x \cdot z$

Suatu near-ring disebut near-ring kanan jika memenuhi aksioma (1), (2), dan (3) bagian (i), dan disebut near-ring kiri jika memenuhi aksioma (1), (2), dan (3) bagian (ii). Selanjutnya yang dimaksud near-ring adalah near-ring kiri, kecuali ada keterangan lebih lanjut, dan $x \cdot y$ dapat juga ditulis $x y$.

Definisi 2. (Clay, [3]) Diberikan near-ring $R$. Subgrup $H$ dari $R$ disebut subnear-ring dari $R$, jika memenuhi $H H \subseteq H$.

Pada near-ring, grupnya tidak harus abelian terhadap operasi + , maka dalam mendefinisikan ideal dari near-ring subgrupnya harus merupakan suatu subrup normal.

Definisi 3. (Clay, [3]) Diberikan near-ring R. Subgrup normal I dari $R$ disebut ideal dari $R$, jika

(1). $R I \subseteq I$

(2). $(r+i) s-r s \in I$ untuk setiap $r, s \in R$ dan $i \in I$.

Subgrup normal $I$ dari $R$, memenuhi kondisi (1) disebut ideal kiri dari $R$, dan memenuhi kondisi (2) disebut ideal kanan dari $R$.

Definisi 4. (Mordeson et al., [5]) Diberikan $X$ adalah himpunan tidak kosong. Suatu pemetaan $\alpha$ disebut subset fuzzy dari $X$ jika $\alpha: X \rightarrow[0,1]$. Selanjutnya himpunan semua subset fuzzy dari $X$ dinotasikan dengan $\mathbb{F}(X)$.

Definisi 5. (Mordeson et al., [5]) Jika $\alpha, \beta \in \mathbb{F}(X)$, maka untuk setiap $x \in X$ :

(1) $\alpha \subseteq \beta$ jika dan hanya jika $\alpha(x) \leq \beta(x)$,

(2) $(\alpha \bigcap \beta)(x)=\min \{\alpha(x), \beta(x)\}$.

Definisi 6. (Kandasamy, [4]) Diberikan near-ring $R$ dan $\alpha \in \mathbb{F}(R)$. Subset fuzzy $\alpha$ disebut subnear-ring fuzzy dari $R$ jika untuk setiap $x, y \in R$ berlaku:

$$
\alpha(x-y) \geq \min \{\alpha(x), \alpha(y)\}, \operatorname{dan} \alpha(x y) \geq \min \{\alpha(x), \alpha(y)\} .
$$

Definisi 7. (Kandasamy, [4]) Diberikan near-ring $R$ dan $\alpha \in \mathbb{F}(R)$. Subset fuzzy $\alpha$ disebut ideal fuzzy dari $R$, jika untuk setiap $x, y, z \in R$ berlaku:

(1) $\alpha(x-y) \geq \min \{\alpha(x), \alpha(y)\}$,

(2) $\alpha(x)=\alpha(y+x-y)$, 
(3) $\alpha(x y) \geq \alpha(y)$, dan

(4) $\alpha((x+z) y-x y) \geq \alpha(z)$.

Suatu $\mu$ disebut ideal kiri fuzzy dari $R$ jika memenuhi kondisi (1), (2), dan (3), sedangkan $\mu$ disebut ideal kanan fuzzy dari $R$ jika memenuhi kondisi (1), (2), dan (4).

Lemma 8. (Abdurrahman et al., [1]) Diberikan near-ring R. Jika a adalah subnear-ring fuzzy dari $R$, maka $\alpha\left(0_{R}\right) \geq \alpha(x)$, dan $\alpha(-x)=\alpha(x)$ untuk setiap $x \in R$.

Definisi 9. (Abou-Zaid, [2]) Diberikan near-ring $R$, dan $\alpha, \beta \in \mathbb{F}(R)$. Penjumlahan $\alpha$ dan $\beta$ didefinisikan dengan,

$$
(\alpha \oplus \beta)(x):= \begin{cases}\sup [\min \{\alpha(y), \beta(z)\}], & x=y+z \\ 0, & x \neq y+z\end{cases}
$$

untuk setiap $x \in R$.

Setelah diberikan definisi, lemma, dan teorema yang mendukung pada pembahasan hasil dari penjumlahan dari subnear-ring fuzzy, dan hasil penjumlahan dari ideal fuzzy near-ring, berikut disajikan lemma dan teorema yang menjadi bahasan dalam tulisan ini.

Lemma 10. Jika $\alpha$ dan $\beta$ adalah subnear-ring fuzzy dari near-ring $R$, maka $(\alpha \oplus \beta)\left(0_{R}\right) \geq(\alpha \oplus \beta)$ $(x)$, dan $(\alpha \oplus \beta)(-x)=(\alpha \oplus \beta)(x)$ untuk setiap $x \in R$.

\section{Bukti:}

Diambil sebarang $x \in \mathrm{R}$, maka $\mathrm{x}=\mathrm{y}+\mathrm{z}$ untuk suatu $\mathrm{y}, \mathrm{z} \in R$. Mengingat $\alpha$ dan $\beta$ adalah subnear ring fuzzy dari $R$, maka menurut Lemma 8: $\alpha\left(0_{R}\right) \geq \alpha(y), \beta\left(0_{R}\right) \geq \beta(z)$, dan $\beta(x)=\beta(-x)$. Akibatnya:

dan,

$$
(\alpha \oplus \beta)\left(0_{R}\right)=\sup \left[\min \left\{\alpha\left(0_{R}\right), \beta\left(0_{R}\right)\right\}\right] \geq \sup [\min \{\alpha(y), \beta(z)\}=(\alpha \oplus \beta)(x) .
$$

$$
(\alpha \oplus \beta)(x)=\sup \left[\min \left\{\alpha\left(0_{R}\right), \beta(x)\right\}\right]=\sup \left[\min \left\{\alpha\left(0_{R}\right), \beta(-x)\right\}\right]=(\alpha \oplus \beta)(-x)
$$

Teorema 11. Diberikan $\alpha$ dan $\beta$ adalah subnear-ring fuzzy dari near-ring $R$. Jika $\beta$ adalah subgrup normal fuzzy dari $R$, maka $\alpha \oplus \beta$ adalah subnear-ring fuzzy dari $R$.

\section{Bukti:}

Diambil sebarang $x, y \in R$ dengan $x=a+b$ dan $y=c+d$ untuk suatu $a, b, c, d \in R$, maka:

$$
\begin{aligned}
(\alpha \oplus \beta)(x-y) & =(\alpha \oplus \beta)(a+b-(c+d))=(\alpha \oplus \beta)(a-c+c+b-c-d) \\
& =\sup [\min \{\alpha(a-c), \beta(c+b-c-d)\}] \\
& \geq \sup [\min \{\min \{\alpha(a), \alpha(c)\}, \min \{\beta(c+b-c), \beta(d)\}\}] \\
& =\sup [\min \{\min \{\alpha(a), \alpha(c)\}, \min \{\beta(b), \beta(d)\}\}] \\
& \geq \min [\sup \{\min \{\alpha(a), \beta(b)\}\}, \sup \{\min \{\alpha(c), \beta(d)\}\}] \\
& =\min \{(\alpha \oplus \beta)(x),(\alpha \oplus \beta)(y)\},
\end{aligned}
$$

dan

$(\alpha \oplus \beta)(x y) \quad=(\alpha \oplus \beta)(x(c+d))=(\alpha \oplus \beta)(x c+x d)=\sup [\min \{\alpha(x c), \beta(x d)\}$

$$
\geq \sup [\min \{\alpha(c), \beta(d)\}]=\min \{(\alpha \oplus \beta)(x),(\alpha \oplus \beta)(y)\} \text {. }
$$

Teorema 12. Jika $\alpha$ dan $\beta$ adalah ideal fuzzy dari near-ring $R$, maka $\alpha \oplus \beta$ adalah ideal fuzzy dari $R$.

\section{Bukti:}

Mengingat $\alpha$ dan $\beta$ adalah ideal fuzzy dari near-ring $R$, maka menurut Teorema 11, berlaku: ( $\alpha$ $\oplus \beta)(x-y) \geq \min \{(\alpha \oplus \beta)(x),(\alpha \oplus \beta)(y)\}$, untuk setiap $x, y \in R$.

Selanjutnya, diambil sebarang $x, y, z \in R$ dengan $x=a+b$, dan $y=c+d$ untuk suatu $a, b, c$, $d \in R$, maka: 


$$
\begin{aligned}
(\alpha \oplus \beta)(y+x-y)= & (\alpha \oplus \beta)(y+a+b-y)=(\alpha \oplus \beta)(y+a-y+y+b-y) \\
= & \sup [\min \{\alpha(y+a-y), \beta(y+b-y)\}] \\
= & \sup [\min \{\alpha(a), \beta(b)\}]=(\alpha \oplus \beta)(x), \text { dan } \\
(\alpha \oplus \beta)[(x+z) y-x y] & =(\alpha \oplus \beta)\left[(x+z) y-x y+0_{R}\right] \\
& =\sup \left[\min \left\{\alpha[(x+z) y-x y], \beta\left(0_{R}\right)\right\}\right] \\
& \geq \sup \left[\min \left\{\alpha(z), \beta\left(0_{R}\right)\right]=(\alpha \oplus \beta)(z) .\right.
\end{aligned}
$$

Lemma 13. Diberikan near-ring $R$. Jika $\alpha$ dan $\beta$ adalah ideal fuzzy dari $R$, maka untuk setiap $x, y \in R:$

(1) $(\alpha \oplus \beta)(x+y)=(\alpha \oplus \beta)(y+x)$, dan

(2) $(\alpha \oplus \beta)(x-y)=(\alpha \oplus \beta)\left(0_{R}\right) \operatorname{maka}(\alpha \oplus \beta)(x)=(\alpha \oplus \beta)(y)$.

\section{Bukti:}

(1) Mengingat $\alpha$ dan $\beta$ adalah ideal fuzzy dari $R$, maka menurut Teorema $12, \alpha \oplus \beta$ adalah ideal fuzzy dari $R$.

Diambil sebarang $x, y \in R$, maka $(\alpha \oplus \beta)(z)=(\alpha \oplus \beta)(-x+z+x)$ untuk setiap $x, z \in R$ yang mengakibatkan,

$$
\begin{aligned}
(\alpha \oplus \beta)(x+y) & =(\alpha \oplus \beta)(-x+[x+y]+x)=(\alpha \oplus \beta)([-x+x]+y+x) \\
& =(\alpha \oplus \beta)(y+x),
\end{aligned}
$$

(2) Misalkan $\alpha \oplus \beta$ ideal fuzzy dari $R$ dan $(\alpha \oplus \beta)(x-y)=(\alpha \oplus \beta)\left(0_{R}\right)$ untuk setiap $x, y \in R$.

Diambil sebarang $x, y \in R$, maka

a) $\quad(\alpha \oplus \beta)(x)=(\alpha \oplus \beta)\left(x+0_{R}\right)=(\alpha \oplus \beta)(x+[-y+y])=(\alpha \oplus \beta)([x-y]+y)$

$$
\begin{aligned}
& =(\alpha \oplus \beta)([x-y]-[-y]) \geq \min \{(\alpha \oplus \beta)(x-y),(\alpha \oplus \beta)(-y)\} \\
= & \min \left\{(\alpha \oplus \beta)\left(0_{R}\right),(\alpha \oplus \beta)(y)\right\}=(\alpha \oplus \beta)(y) .
\end{aligned}
$$

b) $\quad(\alpha \oplus \beta)(y)=(\alpha \oplus \beta)(-y)=(\alpha \oplus \beta)\left(0_{R}-y\right)=(\alpha \oplus \beta)([-x+x]-y)$

$=(\alpha \oplus \beta)(-x+[x-y])=(\alpha \oplus \beta)(-x-[-(x-y)])$

$\geq \min \{(\alpha \oplus \beta)(-x),(\alpha \oplus \beta)(-[x-y])\}$

$=\min \{(\alpha \oplus \beta)(x),(\alpha \oplus \beta)(x-y)\}=\min \left\{(\alpha \oplus \beta)(x),(\alpha \oplus \beta)\left(0_{R}\right)\right\}$

$=(\alpha \oplus \beta)(x)$.

Berdasarkan a) dan b), maka $(\alpha \oplus \beta)(x)=(\alpha \oplus \beta)(y)$ untuk setiap $x, y \in R$.

Lemma 14. Diberikan near-ring $R$, dan $\alpha, \beta \in \mathbb{F}(R)$.

1) Jika $\alpha$ dan $\beta$ adalah ideal fuzzy dari $R$, maka $\alpha \bigcap \beta$ ideal fuzzy dari $R$.

2) Jika a ideal kanan fuzzy, dan $\beta$ ideal kiri fuzzy dari $R$, maka $\alpha \oplus \beta \subseteq \alpha \bigcap \beta$.

\section{Bukti:}

1). Mengingat $\alpha$ dan $\beta$ adalah ideal fuzzy dari $R$, maka $\alpha \bigcap \beta$ adalah ideal fuzzy dari $R$, karena untuk setiap $x, y, i \in R$, berlaku:

$$
\text { a). } \begin{aligned}
(\alpha \bigcap \beta)(x-y) & =\min \{\alpha(x-y), \beta(x-y)\} \\
& \geq \min \{\min \{\alpha(x), \alpha(y)\}, \min \{\beta(x), \beta(y)\}\} \\
& =\min \{\alpha(x), \alpha(y), \beta(x), \beta(y)\} \\
& =\min \{\min \{\alpha(x), \beta(x)\}, \min \{\alpha(y), \beta(y)\}\} \\
& =\min \{(\alpha \bigcap \beta)(x),(\alpha \bigcap \beta)(y)\},
\end{aligned}
$$

b). $(\alpha \bigcap \beta)(x)=\min \{\alpha(x), \beta(x)\}=\min \{\alpha(y+x-y), \beta(y+x-y)\}$

$$
=(\alpha \bigcap \beta)(y+x-y) \text {, }
$$

c). $(\alpha \bigcap \beta)(x y)=\min \{\alpha(x y), \beta(x y)\} \geq \min \{\alpha(y), \beta(y)\}=(\alpha \bigcap \beta)(y)$, dan

d). $(\alpha \bigcap \beta)((x+i) y-x y)=\min \{\alpha((x+i) y-x y), \beta((x+i) y-x y)\}$

$$
\geq \min \{\alpha(i), \beta(i)\}=(\alpha \bigcap \beta)(i) .
$$

2) Misalkan $\alpha$ ideal kanan fuzzy, dan $\beta$ ideal kiri fuzzy dari $R$.

Selanjutnya, diambil sebarang $x \in R$, dengan $x=y+z$, untuk suatu $y, z \in R$. 


\section{Kasus 1:}

Jika $(\alpha \oplus \beta)(x)=0$, maka $0=(\alpha \oplus \beta)(x) \leq(\alpha \bigcap \beta)\left(0_{R}\right)$, yang mengakibatkan untuk setiap $\mathrm{x} \in R$, berlaku: $(\alpha \oplus \beta)(x) \leq(\alpha \bigcap \beta)(x)$. Dengan kata lain, $\alpha \oplus \beta \subseteq \alpha \bigcap \beta$.

Kasus 2:

$\operatorname{Jika}(\alpha o \beta)(x)=\sup [\min \{\alpha(y), \beta(z)\}]$.

Mengingat $\alpha \bigcap \beta$ adalah ideal fuzzy, $\beta$ ideal kiri fuzzy, dan $\alpha$ ideal kanan fuzzy dari $R$, maka $(\alpha \bigcap \beta)(x) \geq \min \{\alpha(x), \beta(x)\}, \beta(x)=\beta(y z) \geq \beta(z)$, dan

$\alpha(x)=\alpha(y z)=\alpha\left(\left(0_{R}+y\right) z-0_{R} z\right) \geq \alpha(y)$.

Akibatnya $(\alpha \oplus \beta)(x) \leq \min \{\alpha(x), \beta(x)\} \leq(\alpha \bigcap \beta)(x)$. Dengan kata lain, $\alpha \oplus \beta \subseteq \alpha \bigcap \beta$.

Lemma 15. Diberikan $\alpha$ dan $\beta$ adalah ideal fuzzy dari near-ring R. Jika $\alpha^{*}(x)=\alpha(x)+1-\alpha$ $\left(0_{R}\right)$, untuk setiap $\alpha^{*} \in \mathbb{F}(R)$ dan $x \in R$, maka $(\alpha \oplus \beta)^{*}$ adalah ideal fuzzy dari $R$ dan $(\alpha \oplus \beta) \subseteq(\alpha \oplus$ $\beta)^{*}$.

\section{Bukti:}

Misalkan $\alpha$ dan $\beta$ ideal fuzzy dari $R$ dan $\alpha^{*}, \beta^{*} \in \mathbb{F}(R)$ dimana $\alpha^{*}(x)=\alpha(x)+1-\alpha\left(0_{R}\right)$, dan $\beta^{*}(x)$ $=\beta(x)+1-\beta\left(0_{R}\right)$ untuk setiap $x \in R$. Akan dibuktikan $(\alpha \oplus \beta)^{*}$ ideal fuzzy dari $R$, dan $(\alpha \oplus \beta) \subseteq(\alpha$ $\oplus \beta)^{\star}$.

Mengingat $\alpha$ dan $\beta$ adalah ideal fuzzy dari $R$, maka menurut Teorema $12, \alpha \oplus \beta$ ideal fuzzy dari $R$, sehingga untuk setiap $x, y, z \in R$, berlaku:

1) $(\alpha \oplus \beta)^{*}(x-y)=(\alpha \oplus \beta)(x-y)+1-(\alpha \oplus \beta)\left(0_{R}\right)$

$$
\begin{aligned}
& \geq \min \{(\alpha \oplus \beta)(x),(\alpha \oplus \beta)(y)\}+1-(\alpha \oplus \beta)\left(0_{R}\right) \\
& =\min \left\{(\alpha \oplus \beta)(x)+1-(\alpha \oplus \beta)\left(0_{R}\right),(\alpha \oplus \beta)(y)+1-(\alpha \oplus \beta)\left(0_{R}\right)\right\} \\
& =\min \left\{(\alpha \oplus \beta)^{*}(x),(\alpha \oplus \beta)^{*}(y)\right\} .
\end{aligned}
$$

2) $\quad(\alpha \oplus \beta)^{*}(x y)=(\alpha \oplus \beta)(x y)+1-(\alpha \oplus \beta)\left(0_{R}\right)$

$$
\begin{aligned}
& \geq \min \{(\alpha \oplus \beta)(x),(\alpha \oplus \beta)(y)\}+1-(\alpha \oplus \beta)\left(0_{R}\right) \\
& =\min \left\{(\alpha \oplus \beta)(x)+1-(\alpha \oplus \beta)\left(0_{R}\right),(\alpha \oplus \beta)(y)+1-(\alpha \oplus \beta)\left(0_{R}\right)\right\} \\
& =\min \left\{(\alpha \oplus \beta)^{*}(x),(\alpha \oplus \beta)^{*}(y)\right\} .
\end{aligned}
$$

2) $\quad(\alpha \oplus \beta)^{*}(y+x-y)=(\alpha \oplus \beta)(y+x-y)+1-(\alpha \oplus \beta)\left(0_{R}\right)$

$$
=(\alpha \oplus \beta)(x)+1-(\alpha \oplus \beta)\left(0_{R}\right)=(\alpha \oplus \beta)^{*}(x) .
$$

3) $(\alpha \oplus \beta)^{*}(x y)=(\alpha \oplus \beta)(x y)+1-(\alpha \oplus \beta)\left(0_{R}\right) \geq(\alpha \oplus \beta)(y)+1-(\alpha \oplus \beta)\left(0_{R}\right)$

$$
=(\alpha \oplus \beta)^{*}(y) \text {. }
$$

4) $\quad(\alpha \oplus \beta)^{*}((x+z) y-x y)=(\alpha \oplus \beta)((x+z) y-x y)+1-(\alpha \oplus \beta)\left(0_{R}\right)$

$$
\geq(\alpha \oplus \beta)(z)+1-(\alpha \oplus \beta)\left(0_{R}\right)=(\alpha \oplus \beta)^{*}(z) .
$$

5) Mengingat $(\alpha \oplus \beta)^{*}\left(0_{R}\right)=(\alpha \oplus \beta)\left(0_{R}\right)+1-(\alpha \oplus \beta)\left(0_{R}\right)$, dan $(\alpha \oplus \beta)^{*}\left(0_{R}\right),(\alpha \oplus \beta)\left(0_{R}\right) \in[0,1]$, maka nilai keanggotaan dari $(\alpha \oplus \beta)^{*}\left(0_{R}\right)=1$. Akibatnya $(\alpha \oplus \beta)(x) \leq(\alpha \oplus \beta)\left(0_{R}\right) \leq 1=(\alpha \oplus \beta)^{*}\left(0_{R}\right)$ untuk setiap $x \in R$.

Karena $(\alpha \oplus \beta)\left(0_{R}\right) \leq 1$ dan $(\alpha \oplus \beta)^{*}(x)=(\alpha \oplus \beta)(x)+1-(\alpha \oplus \beta)\left(0_{R}\right)$, maka $(\alpha \oplus \beta)(x) \leq(\alpha \oplus \beta)^{*}$ $(x)$ untuk setiap $x \in R$, yang mengakibatkan $(\alpha \oplus \beta) \subseteq(\alpha \oplus \beta)^{*}$.

Berdasarkan analisis di atas, maka $(\alpha \oplus \beta)^{\star}$ adalah ideal fuzzy dari $R$, dan $(\alpha \oplus \beta) \subseteq(\alpha \oplus \beta)^{*}$.

Lemma 16. (Swamy et al., [8]) Diberikan near-ring R. Jika $\alpha_{1}, \alpha_{2}, \ldots, \alpha_{n}$ adalah ideal fuzzy dari $R$, maka $\alpha_{1} \oplus \alpha_{2} \oplus \ldots \oplus \alpha_{n}$ adalah ideal fuzzy dari $R$.

\section{Bukti:}

Misalkan $\alpha_{1}, \alpha_{2}, \ldots, \alpha_{n}$ adalah ideal fuzzy dari near-ring $R$. Akan dibuktikan $\alpha_{1} \oplus \alpha_{2} \oplus \ldots \oplus \alpha_{n}$ ideal fuzzy dari $R$.

Untuk membuktikan $\alpha_{1} \oplus \alpha_{2} \oplus \ldots \oplus \alpha_{n}$ adalah ideal fuzzy dari $R$, digunakan induksi matematika pada $\mathrm{n} \geq 2$.

1). Untuk $\mathrm{n}=2$, maka menurut Teorema $12, \alpha_{1} \oplus \alpha_{2}$ adalah ideal fuzzy dari $R$.

2). Asumsikan untuk $n=k, \alpha_{1} \oplus \alpha_{2} \oplus \ldots \oplus \alpha_{k}$ adalah ideal fuzzy dari $R$.

Akan dibuktikan untuk $n=k+1, \alpha_{1} \oplus \alpha_{2} \oplus \ldots \oplus \alpha_{k} \oplus \alpha_{k+1}$ adalah ideal fuzzy dari $R$. 
Mengingat $\alpha_{1} \oplus \alpha_{2} \oplus \ldots \oplus \alpha_{k}$, dan $\alpha_{k+1}$ adalah ideal fuzzy dari $R$, maka menurut Teorema 12 , $\alpha_{1} \oplus \alpha_{2} \oplus \ldots \oplus \alpha_{k} \oplus \alpha_{k}+1$ adalah ideal fuzzy dari $R$.

Dari semua kasus, terbukti bahwa $\alpha_{1} \oplus \alpha_{2} \oplus \ldots \oplus \alpha_{k} \oplus \alpha_{k}+1$ adalah ideal fuzzy dari $R$. Oleh karena itu dengan prinsip induksi matematika, disimpulkan bahwa $\alpha_{1} \oplus \alpha_{2} \oplus \ldots \oplus \alpha_{n}$ adalah ideal fuzzy dari $R$, untuk semua bilangan bulat positif $\mathrm{n}$.

Akibat 17. Diberikan near-ring R. Jika $\alpha_{1}, \alpha_{2}, \ldots, \alpha_{n}$ adalah ideal fuzzy dari $R$, maka $\left(\alpha_{1} \oplus \alpha_{2} \oplus\right.$ $\left.\ldots \oplus \alpha_{n}\right)^{*}$ adalah ideal fuzzy dari $R$.

\section{Simpulan}

Hasil penting atau sifat yang dapat dijadikan sebuah kesimpulan dari tulisan ini adalah penjumlahan dari dua (lebih) ideal fuzzy dari suatu near-ring R, menghasilkan ideal fuzzy dari R.

\section{Daftar Pustaka}

1. Abdurrahman, S., Thresye., Hijriati, N., Ideals fuzzy near-ring, Jurnal Matematika Murni dan Terapan Epsilon, 6(2), 2012, hal 13 - 19.

2. Abou-Zaid, S., On fuzzy subnear-rings and ideals, Fuzzy Sets and Systems, 44(1), 1991, pp. 139-146.

3. Clay, J, R., Nearrings, geneses and applications, Oxford, New York, 1992

4. Kandasamy, W, B, V., Smarandache fuzzy algebra, American Research Press Rehoboth, 2003.

5. Mordeson, J, N., Bhutani, K, R., and Rosenfeld, A., Fuzzy group theory, Springer-Verlag, Berlin Heidelberg, 2005.

6. Pilz, G., Near-ring, the theory and applications $2^{\text {nd }}$ ed, North-Holland Mathematict Studies, vol. 23, North-Holland, Amsterdam, 1983.

7. Satyanarayana, Bh., and Prasad, KS., Near-ring, Fuzzy Ideals, and Graph Theory, Taylor and Francis Group, LLC, 2013.

8. Swamy, P, N., Kumar, K, V., Nagaiah, T., and Srinivas, T., Sum of fuzzy ideals of $\Gamma$-nearrings, Annals of Fuzzy Mathematics and Informatics, x(x), pp. 1-11, 2010. 